\title{
Yukawa unification in SO(10) with light sparticle spectrum
}

\author{
Marcin Badziak, ${ }^{a, b}$ Marek Olechowski ${ }^{c}$ and Stefan Pokorski ${ }^{c}$ \\ ${ }^{a}$ Department of Applied Mathematics and Theoretical Physics, Centre for Mathematical Sciences, \\ University of Cambridge, \\ Wilberforce Road, Cambridge CB3 OWA, U.K. \\ ${ }^{b}$ Cavendish Laboratory, University of Cambridge, J.J. Thomson Avenue, \\ Cambridge CB3 OHE, U.K. \\ ${ }^{c}$ Institute of Theoretical Physics, University of Warsaw \\ ul. Hoża 69, PL-00-681 Warsaw, Poland \\ E-mail: M.Badziak@damtp.cam.ac.uk, Marek.0lechowski@fuw.edu.pl, \\ Stefan.Pokorski@fuw.edu.pl
}

ABSTRACT: We investigate supersymmetric SO(10) GUT model with $\mu<0$. The requirements of top-bottom-tau Yukawa unification, correct radiative electroweak symmetry breaking and agreement with the present experimental data may be met when the soft masses of scalars and gauginos are non-universal. We show how appropriate nonuniversalities can easily be obtained in the $\mathrm{SO}(10)$ GUT broken to the Standard Model. We discuss how values of $\operatorname{BR}(b \rightarrow s \gamma)$ and $(g-2)_{\mu}$ simultaneously in a good agreement with the experimental data can be achieved in $\mathrm{SO}(10)$ model with $\mu<0$. In the region of the parameter space preferred by our analysis there are two main mechanisms leading to the LSP relic abundance consistent with the WMAP results. One is the co-annihilation with the stau and the second is the resonant annihilation via exchange of the $Z$ boson or the light Higgs scalar. A very interesting feature of $\mathrm{SO}(10)$ models with negative $\mu$ is that they predict relatively light sparticle spectra. Even the heaviest superpartners may easily have masses below $1.5 \mathrm{TeV}$ in contrast to multi- $\mathrm{TeV}$ particles typical for models with positive $\mu$.

KEYWORDS: Supersymmetry Phenomenology

ARXIV EPRINT: 1107.2764 


\section{Contents}

1 Introduction 1

2 SO(10) Yukawa unification with negative $\mu \quad 3$

3 Patterns of soft terms allowed in $\mathrm{SO}(10)$ models 5

$4 \quad$ Interplay between $\mathrm{BR}(b \rightarrow s \gamma)$ and $(g-2)_{\mu} \quad 7$

4.1 MSSM contributions to $(g-2)_{\mu} \quad 8$

4.2 MSSM contributions to $\mathrm{BR}(b \rightarrow s \gamma) \quad 9$

$\begin{array}{llr}5 & \text { Neutralino relic abundance } & 11\end{array}$

6 Numerical results $\quad 12$

$\begin{array}{lll}\text { 6.1 Predictions for the MSSM spectrum } & 17\end{array}$

$\begin{array}{llr}7 & \text { Conclusions } & 20\end{array}$

\section{Introduction}

Supersymmetric Grand Unified Theory (SUSY GUT) based on the $\mathrm{SO}(10)$ gauge group is arguably one of the most attractive theories which go beyond the Standard Model (SM). In the simplest version of $\mathrm{SO}(10)$ SUSY GUT, the Minimal Supersymmetric Standard Model (MSSM) Higgs doublets, $H_{u}$ and $H_{d}$, sit in one 10-dimensional representation, while all fermions (together with the right-handed neutrino) of each generation belong to one 16-dimensional representation. In such model not only the gauge couplings but also the Yukawa couplings of top, bottom and tau unify at the GUT scale. This condition together with the requirement of radiative electroweak symmetry breaking (REWSB) strongly restricts the allowed pattern of soft supersymmetry breaking terms. In particular, top-bottom-tau Yukawa unification cannot be obtained if the universal boundary conditions for the soft terms are assumed at the GUT scale [1].

One possibility that makes the top-bottom-tau Yukawa unification viable is some splitting between the soft masses of the Higgs doublets and/or among the third generation squarks [2]. Since $H_{u}$ and $H_{d}$ are parts of the same $\mathrm{SO}(10)$ multiplet, Higgs masses cannot be split by hand. The same applies to sfermions of each generation. However, splitting within $\mathrm{SO}(10)$ multiplets can be consistent with $\mathrm{SO}(10)$ gauge symmetry if $D$-term contributions to the scalar masses are taken into account. Such contributions are generically present in effective theories resulting from $\mathrm{SO}(10)$ GUT models [3] and they simultaneously split soft masses of Higgses, squarks and sleptons. 
Requirement of top-bottom-tau Yukawa unification prefers negative values of the Higgs-mixing parameter, $\mu$, (negative relative to the $\mathrm{SU}(3)$ gaugino mass, $M_{3}$ ) because in such a case the MSSM threshold correction to the bottom quark mass has generically sign appropriate for bottom-tau Yukawa unification. Indeed, it was shown in $[4,5]$ that for $\mu<0$ top-bottom-tau Yukawa unification can be obtained with universal gaugino masses and $D$-term splitting of scalar masses. However, that model predicts a negative SUSY contribution to the muon anomalous magnetic moment, $(g-2)_{\mu}$, and enlarges the observed discrepancy between theory and experiment. This problem can be solved if the $\mathrm{SU}(2)$ gaugino mass, $M_{2}$, is negative.

In this article, we investigate models with negative $\mu$ and non-universal soft masses of scalars and gauginos consistent with $\mathrm{SO}(10)$ symmetry. First, we split the soft scalar masses at the GUT scale using $D$-terms appearing when $\mathrm{SO}(10)$ is broken to the SM gauge symmetry group. We do not introduce any intergenerational splitting at the GUT scale. Second, we assume that the gaugino masses are generated by an $F$-term which is a nonsinglet of $\mathrm{SO}(10)$ transforming as 24-dimensional representation of $\mathrm{SU}(5) \supset \mathrm{SO}(10)$. This assumption results in the following pattern of the gaugino masses: $M_{1}: M_{2}: M_{3}=-1$ : $-3: 2[6]$, which implies that the SUSY contribution to $(g-2)_{\mu}$ is positive, as preferred by the experimental data. We should stress here that even though the gaugino masses are non-universal, there is only one free parameter in this sector which sets the overall scale.

We show that in the above framework top-bottom-tau Yukawa unification can be achieved. We identify some correlations between the GUT scale values of the soft supersymmetry breaking parameters which are required to keep the SUSY threshold correction to the bottom mass small enough to be consistent with Yukawa unification. Given that these correlations hold, Yukawa-unified solutions exist for a very wide range of the gaugino and scalar masses. The main constraints on this model follow from the experimental bounds on $\mathrm{BR}(b \rightarrow s \gamma),(g-2)_{\mu}$ and the dark matter relic abundance. We find that the combination of these constraints, together with the condition of top-bottom-tau Yukawa unification, leads to a rather definite prediction for the SUSY spectrum. In particular, given that $\operatorname{BR}(b \rightarrow s \gamma)$ and $(g-2)_{\mu}$ are compatible with the experimental data at the $2 \sigma$ level, the gluino mass is predicted to be between 500 and $700 \mathrm{GeV}$ or between $900 \mathrm{GeV}$ and $1.6 \mathrm{TeV}$, while all other sparticles have masses below $2 \mathrm{TeV}$. This is the first $\mathrm{SO}(10)$ model in which top-bottom-tau Yukawa unification is consistent with such relatively light SUSY spectrum.

Some aspects of top-bottom-tau Yukawa unification in supersymmetric models has been investigated before. It has been shown [7-9] that the precise unification cannot be obtained with $D$-term splitting of scalar masses if $\mu$ is positive. The level of Yukawa unification improves somewhat if at the GUT scale the third generation scalar masses are different than the scalar masses of the first and the second generation and/or the effect of right-handed neutrinos on the renormalization group equations is taken into account. However, even in such a case the remaining discrepancy [10] may be hard to explain by GUT scale threshold corrections to the Yukawa couplings. On the other hand, Yukawa-unified solutions for $\mu>0$ were found if only Higgs masses were split and soft masses of squarks and sleptons remained universal at GUT scale [7-9]. Beside the fact that this kind of splitting 
explicitly breaks the $\mathrm{SO}(10)$ symmetry, that scenario suffers also from phenomenological drawbacks. Namely, it predicts multi-TeV scalar masses and thermal relic abundance of neutralinos several orders of magnitude larger than the upper experimental bound.

Models with $\mu<0$ may be consistent with the constraints on $\mathrm{BR}(b \rightarrow s \gamma)$ and $(g-$ $2)_{\mu}$ only if $M_{2}$ is negative [11]. This observation ${ }^{1}$ was recently used in [14] where topbottom-tau Yukawa unification was considered in the context of supersymmetric $\mathrm{SU}(4)_{c} \times$ $\mathrm{SU}(2)_{L} \times \mathrm{SU}(2)_{R}$. It was found that assuming $\mu<0$ and $M_{2}<0$ and varying $M_{2}$ and $M_{3}$ independently, top-bottom-tau Yukawa unification can be obtained without a conflict with any experimental data. However, in [14] an ad-hoc Higgs mass splitting was used which in the case of $\mathrm{SO}(10)$, considered in this paper, has no theoretical justification.

The paper is organized as follows: The importance of the sign of $\mu$ on the Yukawa unification is discussed in section 2. Possible patterns of non-universalities of the soft terms are presented in section 3. Section 4 contains a detailed discussion of the MSSM contributions to $\operatorname{BR}(b \rightarrow s \gamma)$ and $(g-2)_{\mu}$. The impact of the experimental bounds on these two quantities on the SUSY parameter space is also analyzed. Some important facts concerning the relic abundance of the neutralinos are recalled in section 5. Section 6 is devoted to the results obtained by our numerical analysis. Finally, we conclude in section 7 .

\section{$2 \mathrm{SO}(10)$ Yukawa unification with negative $\mu$}

Due to the large mass of the top quark, the top-bottom Yukawa unification requires a large value of $\tan \beta \sim m_{t} / m_{b}$. This in turn has a crucial impact on the bottom-tau Yukawa unification because for large $\tan \beta$ there are sizable supersymmetric corrections to the bottom quark mass $[1,15,16]$ :

$$
m_{b}^{\mathrm{MSSM}}=m_{b}^{\mathrm{SM}}\left[1+\left(\frac{\delta m_{b}}{m_{b}}\right)\right]^{-1} .
$$

Unification of the bottom and tau Yukawa couplings requires a negative correction to the bottom mass. The logarithmic correction is positive and of order $\mathcal{O}(5 \%)$. Therefore, the finite correction has to be necessarily negative and large enough to compensate the logarithmic one. The main correction originates from gluino-sbottom and chargino-stop loops $[1,15,16]$ :

$$
\left(\frac{\delta m_{b}}{m_{b}}\right)^{\text {finite }} \approx \frac{g_{3}^{2}}{6 \pi^{2}} \mu m_{\tilde{g}} \tan \beta I\left(m_{\tilde{b}_{1}}^{2}, m_{\tilde{b}_{2}}^{2}, m_{\tilde{g}}^{2}\right)+\frac{h_{t}^{2}}{16 \pi^{2}} \mu A_{t} \tan \beta I\left(m_{\tilde{t}_{1}}^{2}, m_{\tilde{t}_{2}}^{2}, \mu^{2}\right),
$$

where the loop integral $I(x, y, z)$ is defined e.g. in the appendix of [15]. What is relevant for us is that this integral scales as the inverse of the mass squared of the heaviest particle propagating in the loop and it can be approximated by $I(x, y, z) \approx a / \max (x, y, z)$ with

\footnotetext{
${ }^{1}$ Top-bottom-tau Yukawa unification in a model with $\mu<0$ and non-universal gaugino masses assumed to be generated by an $F$-term in 54-dimensional representation of $\mathrm{SO}(10)$ was investigated before in [12]. However, the gaugino mass relation used in that analysis was based on the results of [13] which are incorrect, as was pointed out recently in [6]. Moreover, the Higgs splitting in the model of [12] was introduced ad-hoc rather than by a $D$-term contribution.
} 
$a$ typically between 0.5 and 1 . The numerical coefficient in front of the gluino-sbottom contribution is significantly larger than the one in front of the chargino-stop contribution, so the former contribution dominates in the vast majority of the parameter space. The gluino-sbottom contribution to the bottom mass (2.2) has the same sign as the sign of the $\mu$ parameter. $^{2}$ Therefore, Yukawa unification prefers the negative sign of $\mu$.

Since the gluino-sbottom correction to the bottom mass is the dominant one let us discuss it in some more detail. It is convenient to rewrite it using the experimental value of the strong coupling constant, the properties of the loop integral $I(x, y, z)$ appearing in $(2.2)$ and the value of $\tan \beta \approx 50$ (as required by top-bottom unification):

$$
\left(\frac{\delta m_{b}}{m_{b}}\right)^{\tilde{g} \tilde{b}} \approx \mathcal{O}(0.5-1) \frac{\mu}{m_{\tilde{g}}} \min \left(1,\left(\frac{m_{\tilde{g}}}{m_{\tilde{b}}}\right)^{2}\right),
$$

where $m_{\tilde{b}}$ is the mass of the heavier sbottom. The magnitude of the finite threshold corrections required for bottom-tau unification must be about $10 \%$ to $20 \%$ [17]. From eq. (2.3) it is clear that the correction to the bottom mass is far too large unless $|\mu|<m_{\tilde{g}}$ or $m_{\tilde{g}}<m_{\tilde{b}}$. In the case of $m_{\tilde{g}} \geqslant m_{\tilde{b}}$ one easily finds the following (conservative) upper bound for $|\mu|$ :

$$
|\mu| \lesssim 0.4 m_{\tilde{g}} \approx M_{3}
$$

where $M_{3}$ is the gluino mass at the GUT scale. This bound is relaxed for $m_{\tilde{g}}<m_{\tilde{b}}$ and e.g. $|\mu|$ can be larger than $m_{\tilde{g}}$ for the gluino mass about two times smaller than the heavier sbottom mass.

Yukawa coupling unification may be achieved also for positive $\mu$. However, in such a case, the parameters must satisfy some quite strong constraints. First: the gluinosbottom contribution in (2.2) has the "wrong" sign, so it should be suppressed. This usually requires very large soft sfermion masses $m_{16} \gg M_{1 / 2}$. Second: the soft trilinear parameter $A_{t}$ must be negative and large enough to produce a negative correction to the bottom mass compensating the positive (logarithmic and gluino-sbottom) contributions. This requires the GUT scale parameter $A_{0}$ to be negative, large and carefully chosen (typically $A_{0} \approx-\mathcal{O}(2.5) m_{16}$ ). This characteristic pattern of large soft parameters required by Yukawa unification with positive $\mu$ was found and discussed in $[7,8]$.

Of course, there are some constraints on the soft parameters also in the case of negative $\mu$. However, they are weaker than in models with positive $\mu$. For example: for $\mu>0$, the parameter $A_{t}$ must be large and negative while for $\mu<0$ it can be of both signs and with not too large absolute value. The sign and the magnitude of the gluino-sbottom contribution in (2.2) is typically correct for $\mu<0$ and this should not be spoiled by a too large chargino-stop contribution (neither positive nor negative). The soft sfermion mass parameter $m_{16}$ must be very large for $\mu>0$ while there is no such requirement in the case of $\mu<0$. These differences in the constraints have important phenomenological implications. The SUSY spectrum is very heavy in models with positive $\mu$ but, as we show later, may be relatively light for negative $\mu$.

\footnotetext{
${ }^{2}$ We use the sign convention in which the gluino mass parameter $M_{3}$ and the gluino mass $m_{\tilde{g}}$ are positive.
} 
The sign of $\mu$ is important also for other features of $\mathrm{SO}(10)$ models. For example the sign of the dominant SUSY contribution to the muon anomalous magnetic moment, $(g-2)_{\mu}$, is the same as the sign of the product $\mu M_{2}$. This contribution should be positive because the SM prediction for $(g-2)_{\mu}$ is significantly smaller than the experimentally measured value. Thus, extentions of the SM with a negative contribution to $(g-2)_{\mu}$ are very strongly disfavored. This is the case e.g. for SUSY models with negative $\mu$ and universal gaugino masses. So, in phenomenologically acceptable models with $\mu<0$ one needs gaugino masses with negative $M_{2}$. It will be shown in the next section that this situation can be realized in $\mathrm{SO}(10)$ GUT models.

It is well-known that for universal soft SUSY breaking terms at the GUT scale topbottom-tau Yukawa unification is incompatible with the radiative electroweak symmetry breaking [1]. The main reason is that at the electroweak scale the soft Higgs masses must satisfy the condition

$$
m_{H_{d}}^{2}-m_{H_{u}}^{2}>M_{Z}^{2}
$$

For large $\tan \beta$ and heavy top quark, the value of $M_{1 / 2}^{2}$ is strongly correlated with that of $\mu^{2}$. This correlation implies $\mu^{2}>M_{1 / 2}^{2}$ which is inconsistent with the upper bound (2.4) on $|\mu|$ and leads to a too large (positive or negative) correction to the bottom mass. As a result, the correct REWSB can not be achieved in $\mathrm{SO}(10)$ models with universal soft terms at the GUT scale.

The situation changes when non-universalities are allowed. Patterns of non-universal scalar masses which make top-bottom-tau Yukawa unification compatible with the REWSB were identified in [2]. Two examples of such patterns at the GUT scale are: $m_{H_{d}}^{2}>m_{H_{u}}^{2}>$ $m_{0}^{2}$ and $m_{D}^{2}<m_{U}^{2}<m_{0}^{2}$, where $m_{0}$ is the common value of the soft mass of all particles other than $H_{d}$ and $H_{u}(D$ and $U$ ) in the first (second) case. As we will show below, similar non-universalities may very naturally emerge in the SO(10) GUT models.

\section{Patterns of soft terms allowed in $\mathrm{SO}(10)$ models}

$\mathrm{SO}(10)$ GUT is a very predictive theory since its structure allows only few free parameters. Both Higgs doublets reside in the same representation so they have a common soft SUSY breaking mass, $m_{10}$. The same happens with all squarks and sleptons of one generation and we denote their mass as $m_{16}$ which, in general, can be different from $m_{10}$ (there can be three different soft sfermion masses, one for each generation, but we do not consider intergenerational splitting in this paper). In addition, in the effective MSSM (below the GUT scale) there is another source of scalar masses which may differentiate masses within each $\mathrm{SO}(10)$ representation. It originates from breaking of $\mathrm{U}(1)$ which occurs when $\mathrm{SO}(10)$ is broken down to SM gauge group. Even though the exact mechanism of GUT symmetry breaking is unknown, it is plausible that it occurs due to the existence of some SM singlets which are charged under the additional $\mathrm{U}(1)$ (which is part of $\mathrm{SO}(10)$ ) and acquire vacuum expectation values. Since masses of these SM singlets are expected to be around the GUT scale, they can be integrated out. It can be shown that in the effective theory the MSSM scalars acquire squared mass corrections proportional to their charges under the broken $\mathrm{U}(1)$ [3]. The magnitude of these corrections is set by the $D$-term of the additional U(1). 
Taking this into account one finds the following generic structure of scalar masses in $\mathrm{SO}(10)$ models:

$$
\begin{aligned}
m_{H_{d}}^{2} & =m_{10}^{2}+2 D, \\
m_{H_{u}}^{2} & =m_{10}^{2}-2 D, \\
m_{Q, U, E}^{2} & =m_{16}^{2}+D, \\
m_{D, L}^{2} & =m_{16}^{2}-3 D,
\end{aligned}
$$

where $D$ parameterizes the size of a $D$-term contribution. It was demonstrated in [18] that the above source of non-universal scalar masses, for a certain range of the parameters $m_{16}$ and $m_{10}$, can lead to top-bottom Yukawa unification in agreement with REWSB if $D$ is positive. ${ }^{3}$ However, such non-universalities are not enough if one wants to construct models satisfying all present experimental constraints.

In GUT models the gaugino masses are usually assumed to be universal. However, they are universal only in the special case when the SUSY breaking $F$-term which gets a vacuum expectation value is a singlet of the GUT gauge symmetry group. In general, the gaugino masses in supergravity can arise from the following dimension five operator:

$$
\mathcal{L} \supset-\frac{F^{a b}}{2 M_{\text {Planck }}} \lambda^{a} \lambda^{b}+\text { c.c. },
$$

where $\lambda^{a}$ are the gaugino fields. The resulting gaugino mass matrix is $\frac{\left\langle F^{a b}\right\rangle}{M_{\text {Planck }}}$. The vacuum expectation value of the relevant $F$-term, $\left\langle F^{a b}\right\rangle$, must transform as the singlet of the SM gauge group but it can be a non-singlet of the full GUT group. Since the gauginos belong to the adjoint representation, non-zero gaugino masses may arise from VEVs of the $F$-terms transforming as any of the representations present in the symmetric part of the direct product of the two adjoints, which for $\mathrm{SO}(10)$ reads:

$$
(45 \times 45)_{\mathrm{S}}=1+54+210+770 .
$$

If SUSY is broken by an F-term transforming as a non-singlet representation of $\mathrm{SO}(10)$, gaugino masses are not universal. The classification of non-universal gaugino masses for $\mathrm{SO}(10)$ and its subgroups was provided in [6].

From our point of view the most interesting case is when SUSY is broken by an $F$-term transforming as the 24-dimensional representation of $\mathrm{SU}(5) \supset \mathrm{SO}(10)$. Such $F$-term gives a negative contribution to the gauginos associated with the $\mathrm{SU}(2)$ subgroup of the $\mathrm{SM}$ gauge group:

$$
M_{1}: M_{2}: M_{3}=-\frac{1}{2}:-\frac{3}{2}: 1,
$$

where $M_{1}, M_{2}$ and $M_{3}$ are the bino, wino and gluino masses, respectively. The overall scale of gaugino masses, $M_{1 / 2}=M_{3}$, is treated as a free parameter.

\footnotetext{
${ }^{3}$ For positive $D$, one gets $m_{H_{d}}^{2}>m_{H_{u}}^{2}$ and $m_{D}^{2}<m_{U}^{2}$ as in two patterns of non-universalities proposed in [2] (mentioned after eq. (2.5)). In [18], the parameters $m_{16}$ and $m_{10}$ were taken as universal at the Planck scale and their values at the GUT scale were obtained by RG running in $\mathrm{SO}(10)$. In the present paper, we take them as free parameters.
} 
The 24-dimensional representation of $\mathrm{SU}(5)$ appears in each of the three non-singlet representations of $\mathrm{SO}(10)$ in the r.h.s. of eq. (3.3). However, the most economical choice is the $\mathbf{5 4}$ representation. It is the smallest one and it contains only one SM singlet (in $\mathbf{2 4}$ of SU(5)). Representations $\mathbf{2 1 0}$ and $\mathbf{7 7 0}$ contain 3 and 4 SM singlets, respectively.

In this paper, we assume that the gaugino masses arise predominantly from the $F$-term in $\mathbf{5 4}$ representation (or a part of $\mathbf{2 1 0}$ or $\mathbf{7 7 0}$ representation transforming as $\mathbf{2 4}$ of SU(5)) so they satisfy the relation (3.4).

For simplicity, we assume that soft scalar masses are given by (3.1) and the soft trilinear terms have a universal value $A_{0}$. The first assumption is realized when the non-singlet $F$ term appears in the soft scalar terms only in the singlet combination (e.g. the singlet in the product $\mathbf{5 4} \times \mathbf{5 4}$ ). The latter assumption requires the existence of a singlet F-term, in addition to a non-singlet one. Such singlet must dominate the trilinear terms and may contribute to the scalar masses but should give only a subdominant contribution to the gaugino masses. The consequences of dropping these two simplifying assumptions will be discussed elsewhere.

\section{Interplay between $\mathrm{BR}(b \rightarrow s \gamma)$ and $(g-2)_{\mu}$}

The experimental bounds on two quantities: $\operatorname{BR}(b \rightarrow s \gamma)$ and $(g-2)_{\mu}$, give quite strong constraints on the SUSY extensions of the SM. The reason is as follows: The SM prediction [19] for the anomalous muon magnetic moment is more than $3 \sigma$ below the present experimental result [20]:

$$
\begin{aligned}
& a_{\mu} \stackrel{\text { SM }}{=}(11659180.2 \pm 4.9) \times 10^{-10}, \\
& a_{\mu} \stackrel{\exp }{=}(11659208.9 \pm 6.3) \times 10^{-10},
\end{aligned}
$$

where $a_{\mu} \equiv(g-2)_{\mu} / 2$. Combining in quadrature theoretical and experimental errors yields the following result for the discrepancy between experiment and the SM prediction:

$$
\delta a_{\mu} \equiv a_{\mu}^{\exp }-a_{\mu}^{\mathrm{SM}}=(28.7 \pm 8.0) \times 10^{-10} .
$$

Therefore, the SUSY contribution to $(g-2)_{\mu}$ should be rather big and necessarily positive. On the other hand, the SM prediction [21] for $\mathrm{BR}(b \rightarrow s \gamma)$ is quite close to the experimental result [22]:

$$
\begin{aligned}
& \mathrm{BR}^{\mathrm{SM}}(b \rightarrow s \gamma)=(3.15 \pm 0.23) \times 10^{-4}, \\
& \operatorname{BR}^{\exp }(b \rightarrow s \gamma)=(3.55 \pm 0.24 \pm 0.09) \times 10^{-4},
\end{aligned}
$$

leaving no much room for the SUSY contribution. Moreover, the charged Higgs exchange always increases the SM result so the contributions involving superpartner loops must be small or negative.

Typically, SUSY effects decrease with the SUSY mass scale. So, the measured value of $(g-2)_{\mu}$ strongly prefers small $M_{\mathrm{SUSY}}$ while constraints on $\mathrm{BR}(b \rightarrow s \gamma)$ can be easier fulfilled for bigger $M_{\mathrm{SUSY}}$. One should remember that the LEP bound on the Higgs mass also requires heavy superpartners (at least the stops). This, apparently, leads to a tension between different experimental results. 
It should be stressed that one should be very careful when using $(g-2)_{\mu}$ and $\mathrm{BR}(b \rightarrow$ $s \gamma)$ to exclude various MSSM scenarios. On the one hand, there are still unresolved issues concerning the calculation of $(g-2)_{\mu}$ in the Standard Model (such as the value of the hadronic contribution) and it may eventually turn out that the observed discrepancy between the SM and the experiment is not so big. On the other hand, the calculation of $\mathrm{BR}(b \rightarrow s \gamma)$ in the MSSM may be affected by some non-minimal flavour violating effects such as non-vanishing off-diagonal elements of soft mass squared matrix for the up-type squarks (see e.g. [23, 24]). Keeping this in mind, we will first consider separately two cases in which only one of the two above mentioned constraints is imposed on our $\mathrm{SO}(10)$ model. Then, we will focus on the question if both constraints can be satisfied simultaneously. In the following subsections we discuss the main MSSM contribution to $(g-2)_{\mu}$ and $\operatorname{BR}(b \rightarrow s \gamma)$ in some more detail.

\subsection{MSSM contributions to $(g-2)_{\mu}$}

Two dominant contributions to $(g-2)_{\mu}$ in the MSSM originate from the one-loop diagrams involving charginos accompanied by the muon sneutrino and neutralinos accompanied by smuons. The $\tan \beta$ enhanced part of the chargino-sneutrino contribution is given by $[25,26]$ :

$$
a_{\mu}^{\chi^{ \pm}} \approx \frac{g_{2} h_{\mu} m_{\mu}}{24 \pi^{2}} \sum_{a} \frac{m_{\chi_{a}^{+}}}{m_{\tilde{\nu}_{\mu}}^{2}} U_{a 2} V_{a 1} F_{2}^{C}\left(\frac{m_{\chi_{a}^{+}}^{2}}{m_{\tilde{\nu}_{\mu}}^{2}}\right),
$$

where the loop function $F_{2}^{C}$ may be found in $[25,26] .{ }^{4}$ Assuming that all SUSY particles are degenerate with a common mass $M_{\mathrm{SUSY}}$ and neglecting terms proportional to $M_{W} / M_{\mathrm{SUSY}}$, the chargino contribution may be further approximated as

$$
a_{\mu}^{\chi^{ \pm}} \approx \frac{1}{32 \pi^{2}} \frac{m_{\mu}^{2}}{M_{\mathrm{SUSY}}^{2}} g_{2}^{2} \operatorname{sgn}\left(\mu M_{2}\right) \tan \beta .
$$

In the same approximation the neutralino-smuon contribution reads

$$
a_{\mu}^{\chi^{0}} \approx \frac{1}{192 \pi^{2}} \frac{m_{\mu}^{2}}{M_{\mathrm{SUSY}}^{2}}\left[g_{1}^{2} \operatorname{sgn}\left(\mu M_{1}\right)-g_{2}^{2} \operatorname{sgn}\left(\mu M_{2}\right)\right] \tan \beta .
$$

The numerical coefficient in front of the chargino contribution (4.5) is several times bigger than the one in front of the neutralino contribution (4.6). Thus, typically the chargino loop dominates the SUSY contribution to $a_{\mu}$. Its value (4.4) decreases with $m_{\tilde{\nu}_{\mu}}^{2}$, so experimental data on $(g-2)_{\mu}$ favor light muon sneutrino.

Formulae (4.5) and (4.6) are valid in the limit in which all SUSY particles have a common mass $M_{\text {SUSY }}$. Actually different diagrams involve different masses. Thus, in principle the neutralino contribution might be dominant if smuons were much lighter than the muon sneutrino. However, this is not the case in our model because the $D$-term splitting of the scalar masses implies that $\tilde{\mu}_{R}$ is always heavier than the muon sneutrino ( $\tilde{\mu}_{L}$ is almost degenerate with muon sneutrino). In our numerical calculations we use full expressions for $(g-2)_{\mu}$ but to discuss qualitatively the results it is enough to use only the chargino-sneutrino contribution (4.4).

\footnotetext{
${ }^{4} \mathrm{~A}$ factor of $1 / 2$ is missing in the definition of $F_{2}^{C}$ in ref. [26].
} 


\subsection{MSSM contributions to $\mathrm{BR}(b \rightarrow s \gamma)$}

Within MSSM there are two possibly sizable contributions to $\mathrm{BR}(b \rightarrow s \gamma)$. The first one, involving the loop with the charged Higgs boson, always adds constructively to the SM result. The second important contribution comes from the diagrams with charginos and accompanying squarks in the loop. The chargino contribution is enhanced by $\tan \beta$ so it may be especially large in models with top-bottom-tau Yukawa unification considered in this paper.

For large $\tan \beta$ the chargino-squark contribution is dominated by the following part of the relevant Wilson coefficients [27]

$$
\begin{gathered}
C_{7,8}^{\chi^{+} \approx \frac{1}{\cos \beta} \sum_{a=1,2}\{} \frac{U_{a 2} V_{a 1} M_{W}}{\sqrt{2} m_{\chi_{a}^{+}}}\left[F_{7,8}^{(3)}\left(\frac{m_{\tilde{q}}^{2}}{m_{\chi_{a}^{+}}^{2}}\right)-c_{\tilde{t}}^{2} F_{7,8}^{(3)}\left(\frac{m_{\tilde{t}_{1}}^{2}}{m_{\chi_{a}^{+}}^{2}}\right)-s_{\tilde{t}}^{2} F_{7,8}^{(3)}\left(\frac{m_{\tilde{t}_{2}}^{2}}{m_{\chi_{a}^{+}}^{2}}\right)\right] \\
\left.+s_{\tilde{t}} c_{\tilde{t}} \frac{U_{a 2} V_{a 2} m_{t}}{2 \sin \beta m_{\chi_{a}^{+}}}\left[F_{7,8}^{(3)}\left(\frac{m_{\tilde{t}_{1}}^{2}}{m_{\chi_{a}^{+}}^{2}}\right)-F_{7,8}^{(3)}\left(\frac{m_{\tilde{t}_{2}}^{2}}{m_{\chi_{a}^{+}}^{2}}\right)\right]\right\},
\end{gathered}
$$

where $U$ and $V$ are matrices which diagonalize the chargino mass matrix while $s_{\tilde{t}}\left(c_{\tilde{t}}\right)$ denotes the sine (cosine) of the stop mixing angle. For more details and the definition of functions $F_{7,8}^{(3)}$ see for example [27]. We will refer to the contribution in the second line in the above formula as a stop-mixing one because it vanishes for vanishing stop mixing. The sign of this contribution, relative to the SM prediction, is the same as $\operatorname{sgn}\left(\mu A_{t}\right)$. So, it may lead to problems with the $b \rightarrow s \gamma$ branching ratio in models with negative $\mu$ because $A_{t}$ very often is negative due to the RGE running. One possible way out is to have $A_{0}$ a few times larger than $M_{1 / 2}$ at the GUT scale because in such a case $A_{t}$ can be positive at the EW scale. As a result, stop-mixing contribution would be negative as preferred by phenomenology.

Whether $\mathrm{BR}(b \rightarrow s \gamma)$ can satisfy the experimental constraints with light charginos (as preferred by $\left.(g-2)_{\mu}\right)$ and values of $A_{0}$ in a wide range, depends on the sign and the magnitude of the contribution in the first line in (4.7). This contribution will be called the gaugino contribution because it involves the gaugino components of the charginos $V_{a 1}$. Using the definitions of the chargino mixing matrices $U$ and $V$ (see e.g. [28] with an obvious modification for negative $M_{2}$ ) one can find that the sign of this contribution, relative to the SM contribution, is given by $\operatorname{sgn}\left(-\mu M_{2}\right)$. This is very important for the models considered in this paper. We have chosen negative $\mu$ (preferred by the Yukawa unification) and negative $M_{2}$ (required by $(g-2)_{\mu}$ analysis when $\mu<0$ ). As a result, the gaugino part of the chargino-stop contribution to $\mathrm{BR}(b \rightarrow s \gamma$ ) (first line in (4.7)) has the sign opposite to that of the SM and the charged Higgs contributions. This helps to obtain better agreement of the $b \rightarrow s \gamma$ branching ratio with the experimental results.

The gaugino part of the chargino-stop contribution has the "correct" sign preferred by phenomenology. However, it may really help to fulfill the experimental bounds only if its magnitude is not negligible. Let us check when this is the case. The expression in the square bracket in the first line of (4.7) is suppressed by the squark GIM mechanism. Moreover, the first line is suppressed with respect to the second one by the factor $M_{W} / m_{t}$. This last 
suppression is not very strong, a factor of about $1 / 2$, and may easily be compensated by other sources. Also the squark GIM mechanism may be not very efficient when the stop quark masses, $m_{\tilde{t}_{1,2}}$, are substantially smaller than the averaged squark mass for the first and second families, denoted by $m_{\tilde{q}}$.

There are two more important differences between the two parts of eq. (4.7): The second line is proportional to $s_{\tilde{t}} c_{\tilde{t}}$ so its value may be suppressed by a smallness of the stop mixing. In addition, the ratio of those two contributions is proportional to $V_{11} / V_{12}$. This ratio may be bigger than 1 if the lighter chargino $\chi_{1}^{+}$is dominated by the gaugino component which happens when $M_{2}^{2}$ is smaller than $\mu^{2}$. The value of $V_{11} / V_{12}$ increases with that of $\mu^{2} / M_{2}^{2}$ and can be very large. However, one should notice that a very large ratio $\mu^{2} / M_{2}^{2}$ suppresses both contributions because of the common factor $U_{12}$.

Summarizing: the gaugino part of the chargino-stop contribution to $b \rightarrow s \gamma$ (first line in (4.7)) may be comparable to or even more important than the stop-mixing part (second line) if some of the following conditions are met: the stop mixing is small; the stops are much lighter than the other up-type squarks; the lighter chargino is dominated by the gaugino component ( $V_{11}$ substantially bigger than $V_{12}$ ).

It turns out that all three above conditions are typically easier to satisfy in the presence of a hierarchy $M_{1 / 2} \ll m_{16}$. First of all, for $M_{1 / 2} \ll m_{16}$ the RGEs for $m_{Q}$ and $m_{U}$ are dominated by terms proportional to the Yukawa couplings so the mass splitting between the stops and other up-type squarks is maximized. Secondly, since RGE running usually results in $A_{t} \sim \mathcal{O}\left(-M_{1 / 2}\right)$ the hierarchy $M_{1 / 2} \ll m_{16}$ naturally suppresses the stop mixing angle. These arguments are general and are not restricted to models with top-bottom-tau Yukawa unification. On the other hand, the fact that for $M_{1 / 2} \ll m_{16}$ it is easier to obtain the lighter chargino dominated by the gaugino component, is specifically related to the assumption of top-bottom-tau Yukawa unification. This follows from the fact that the requirement of not too large gluino-sbottom correction to the bottom mass (2.3) results in the upper bound for $\left|\mu / M_{2}\right|$ :

$$
\left|\mu / M_{2}\right| \lesssim 0.8 \max \left(1,\left(\frac{m_{\tilde{b}}}{m_{\tilde{g}}}\right)^{2}\right),
$$

where we used the approximate relation $m_{\tilde{g}} \approx 2 M_{2}$ resulting from the one-loop RGEs and the assumed pattern of the gaugino masses (3.4) at the GUT scale. It is clear from the above formula that Yukawa unification implies that the lighter chargino is dominated by the higgsino component unless the gluino is substantially lighter than the heavier sbottom and this is possible only if $M_{1 / 2} \ll m_{16}$.

The above discussed gaugino part of the chargino-stop contribution is very important for our analysis. In the models considered in this paper (negative $\mu$ and $M_{2}$ ) it is negative and so helps to obtain acceptable values of $\mathrm{BR}(b \rightarrow s \gamma)$ for lighter SUSY spectrum. This is very desirable because the experimental data on $(g-2)_{\mu}$ strongly favor light muon sneutrino and charginos. Our numerical calculations show that it is possible to fulfill the experimental bounds simultaneously on $(g-2)_{\mu}$ and $\operatorname{BR}(b \rightarrow s \gamma)$. The detailed discussion is given in section 6 . 


\section{$5 \quad$ Neutralino relic abundance}

Experimental data on $\mathrm{BR}(b \rightarrow s \gamma)$ and $(g-2)_{\mu}$ result in quite strong constraints on SUSY models. Another important information which can be used to restrict the SUSY parameter space comes from the dark matter (DM) relic abundance measured by WMAP collaboration [29]

$$
\Omega_{\mathrm{DM}} h^{2}=0.1120 \pm 0.0056 .
$$

In supersymmetric models with R-parity conservation the lightest supersymmetric particle (LSP), which is very often the neutralino, is stable. In consequence LSP can play the role of DM. In the most desirable situation the LSP could have the relic abundance within the experimental bounds (5.1) and so constitute the dominant component of DM. Less interesting but still acceptable possibility is the LSP relic abundance is below the observed value. The neutralino relic abundance depends crucially on the type of its dominant component. In models considered in this paper the main component of the lightest neutralino is bino. The reason is as follows: Due to the pattern of the gaugino masses at the GUT scale (3.4) and their RG running, the gaugino mass ratio at the EW scale is given roughly by $\left|M_{1}\right|:\left|M_{2}\right|:\left|M_{3}\right| \approx 1: 6: 12$. This implies that wino cannot be the main component of the LSP. Whether bino or higgsino dominates LSP depends on the ratio $\left|\mu / M_{1}\right|$. The requirement of bottom-tau Yukawa unification sets the following (conservative) constraint on this ratio:

$$
1.2 \lesssim\left|\frac{\mu}{M_{1}}\right| \lesssim 5
$$

which follows from the requirement that the sbottom-gluino correction to the bottom mass (2.3) is between $10 \%$ and $20 \%$ and from the approximate relation $m_{\tilde{g}} \approx 12\left|M_{1}\right|$ at the electroweak scale. The above reasoning shows that the LSP is mainly bino but in some cases the higgsino component may be non-negligible.

Unfortunately, for the bino-like LSP, typical neutralino relic abundance is much bigger then (5.1). Approximate analytical formulae for $\Omega_{\mathrm{DM}} h^{2}$ can be found e.g. in [30]. One can identify three situations when the neutralino relic abundance is not too large: First: there are some relatively light superpartners which couple strongly enough to the LSP and to some SM particles. Second: there is a superpartner only slightly heavier than the LSP allowing for effective co-annihilation. Third: there is a particle approximately two times heavier than the LSP coupled strongly enough to the LSP and to some SM particles allowing for a resonance enhancement of the annihilation cross section. None of these three possibilities is very easy to realize. Lower experimental bounds on SUSY particles masses make it more and more difficult to use the first, simplest, way of decreasing the LSP relic abundance. Two other methods, co-annihilation and resonance annihilation, require some sort of tuning the LSP mass with the mass of some other superpartner. So, the condition that the LSP relic abundance is below the upper experimental bound results usually in quite substantial reduction of the allowed part of the SUSY parameter space. Our numerical results show that this is the case also for the models considered in this paper. Quite big parts of the parameter space allowed by all other constrains give too large $\Omega_{\mathrm{DM}} h^{2}$. Nevertheless, we will show that it is possible to fulfill the upper bound on 
the LSP relic abundance. Moreover, each of the three above mentioned mechanism may be used in our model. There are regions in which the neutralino annihilation cross section is large enough due to co-annihilation (mainly with stau), resonance annihilation (via $h^{0}$, $A^{0}$ or $Z$ boson exchange) or presence of light superpartners (e.g. sbottom).

\section{$6 \quad$ Numerical results}

In order to analyze quantitatively our model we solved numerically the 2-loop renormalization group equations implementing proper REWSB and calculated the sparticle spectrum using SOFTSUSY [31] interfaced with MicrOmegas [32] for calculating the relic density of dark matter, as well as, $\operatorname{BR}(b \rightarrow s \gamma),(g-2)_{\mu}$ and $\operatorname{BR}\left(B_{s} \rightarrow \mu^{+} \mu^{-}\right)$. We use the following values of relevant experimental inputs: $m_{t}=173.3 \mathrm{GeV}, m_{b}\left(m_{b}\right)=4.2 \mathrm{GeV}$, $\alpha_{s}\left(M_{Z}\right)=0.1187$.

It is convenient to use the following quantity:

$$
\left.R \equiv \frac{\max \left(h_{t}, h_{b}, h_{\tau}\right)}{\min \left(h_{t}, h_{b}, h_{\tau}\right)}\right|_{\mathrm{GUT}}
$$

to quantify the goodness of Yukawa unification. In a search for solutions with $R$ close to unity we randomly scanned the parameter space defined by:

$$
\begin{array}{lll}
0 \leqslant m_{16} \leqslant 2000 \mathrm{GeV}, & 0 \leqslant M_{1 / 2} \leqslant 2000 \mathrm{GeV}, & 0.1 \leqslant m_{10} / m_{16} \leqslant 2, \\
0 \leqslant D / m_{16}^{2} \leqslant 0.3, & -3 \leqslant A_{0} / m_{16} \leqslant 3, & 40 \leqslant \tan \beta \leqslant 55
\end{array}
$$

and $\mu<0$. The above ranges for the parameters were chosen for the following reasons: First of all, we scanned $m_{16}$ and $M_{1 / 2}$ only below $2 \mathrm{TeV}$ because we are most interested in solutions which predict light enough spectrum which explain the $(g-2)_{\mu}$ anomaly and could be detected at the LHC. Secondly, we scanned only over positive values of $D$ since negative ones seems to be inconsistent with top-bottom-tau Yukawa unification. Large values of $\tan \beta$ are necessary to produce the observed ratio of top to bottom masses. Ranges for the remaining parameters were chosen wide enough not to miss any solutions giving Yukawa unification.

For every randomly generated point we demand proper REWSB and the neutralino being LSP. We also apply the following experimental constraints:

$$
\begin{aligned}
12.7 \cdot 10^{-10} & <\delta a_{\mu}^{\mathrm{SUSY}}<44.7 \cdot 10^{-10} \quad(2 \sigma) \\
2.89 \cdot 10^{-4} & <\mathrm{BR}(b \rightarrow s \gamma)<4.21 \cdot 10^{-4} \\
\mathrm{BR}\left(B_{s} \rightarrow \mu^{+} \mu^{-}\right) & <5.8 \cdot 10^{-8} \\
\Omega_{\mathrm{DM}} h^{2} & <0.1288 \quad(3 \sigma) \\
m_{h^{0}} & >111.4 \mathrm{GeV}
\end{aligned}
$$

and the mass limits on SUSY particles from LEP and Tevatron. In particular, we used the lower bound for the gluino mass $m_{\tilde{g}}>220 \mathrm{GeV}$ [33]. The impact of the LHC searches for SUSY particles will be discussed later on. 
Several comments on the above choice of constraints are in order. We imposed only the upper bound on the relic density as a necessary consistency condition. We will show later a few benchmark solutions for which the WMAP bound on $\Omega_{\mathrm{DM}} h^{2}$ is saturated by the neutralino contribution. Due to the fact that the uncertainty in the prediction of the lightest Higgs boson mass is about $3 \mathrm{GeV}$ [34] we used slightly relaxed LEP2 bound [35]. We use the upper bound on $\operatorname{BR}\left(B_{s} \rightarrow \mu^{+} \mu^{-}\right)$from Tevatron [36]. We imposed the $2 \sigma$ bound on the SUSY contribution to $(g-2)_{\mu}$. So, we demand from the theory to really explain the $(g-2)_{\mu}$ anomaly rather than demand only that the SUSY contribution to $(g-2)_{\mu}$ is positive (to do no worse than the SM) which is often done in the literature. This will allow us to understand better the implications of the $(g-2)_{\mu}$ constraint.

In what follows we present the results of our numerical analysis. As we said earlier, for several reasons it is interesting to check how constraining for the Yukawa coupling unification are separately $(g-2)_{\mu}$ and $\mathrm{BR}(b \rightarrow s \gamma)$ experimental results, as well as the WMAP bound on $\Omega_{\mathrm{DM}} h^{2}$. In figure 1 we present a plot of $R$ vs $m_{16}$. Perfect top-bottom-tau Yukawa unification (i.e. $R=1$ ) can be obtained for $m_{16} \gtrsim 300 \mathrm{GeV}$. The main experimental constraints that restrict possible values of $m_{16}$ are $(g-2)_{\mu}$ and $\mathrm{BR}(b \rightarrow s \gamma)$. For the values of $m_{16}$ between about 250 and $1500 \mathrm{GeV}(g-2)_{\mu}$ may be within the $2 \sigma$ experimental bound and Yukawa unification may be obtained at least at the $10 \%$ level (the region between blue contours in figure 1). On the other hand, Yukawa-unified solutions giving correct values of $\mathrm{BR}\left(b \rightarrow s \gamma\right.$ ) (at the $2 \sigma$ level) are found for $m_{16} \gtrsim 700 \mathrm{GeV}$ (the region to the right of the red contour in figure 1). The most interesting fact which follows from our numerical analysis is that there exists a rather wide range of values of $m_{16}$, between about 700 and $1500 \mathrm{GeV}$, giving a good agreement with the assumption of top-bottom-tau Yukawa unification (the overlapping region of the red and blue contours in figure 1).

The WMAP bound on the relic density of neutralinos does not constrain the values of $m_{16}$ consistent with top-bottom-tau unification. However, this does not mean that the requirement of not too large relic abundance of neutralinos does not constrain the parameter space at all. In figure 2 solutions with top-bottom-tau Yukawa unification at the level of $10 \%$ or better (i.e. $R \leqslant 1.1$ ) are shown in the $m_{16}-M_{1 / 2}$ plane. Without imposing the WMAP bound (6.6) Yukawa-unified solutions are found inside the blue contour if they satisfy the constraint $(6.3)$ on $(g-2)_{\mu}$ and to the right of the red contour if they satisfy the constraint $(6.4)$ on $\mathrm{BR}(b \rightarrow s \gamma)$. In the overlapping region of the blue and red contours constraints on both $(g-2)_{\mu}$ and $\mathrm{BR}(b \rightarrow s \gamma)$ can be satisfied simultaneously. Even though Yukawa unification with acceptable values of $(g-2)_{\mu}$ and $\operatorname{BR}(b \rightarrow s \gamma)$ can be found for a rather wide range of $M_{1 / 2}$ between about 100 and $650 \mathrm{GeV}$, in almost the entire part of the parameter space with $M_{1 / 2}$ below about $350 \mathrm{GeV}$ some overabundance of neutralinos is predicted. Viable solutions with low $M_{1 / 2}$ are found only in two narrow strips around $M_{1 / 2} \approx 200 \mathrm{GeV}$ and $M_{1 / 2} \approx 250 \mathrm{GeV}$ which correspond to the mass of the LSP around 45 and $55 \mathrm{GeV}$, respectively. Such masses result in very efficient resonant annihilation of neutralinos through $Z$ boson or the light CP-even Higgs exchange. As seen from figure 2 these resonances are very narrow if the $b \rightarrow s \gamma$ constraint (6.4) is satisfied. This follows from the fact that the couplings of the LSP to $Z$ and $h^{0}$ grow with increasing Higgsino component of the LSP. Therefore, the resonances are wider for smaller values of $\left|\mu / M_{1}\right|$. 


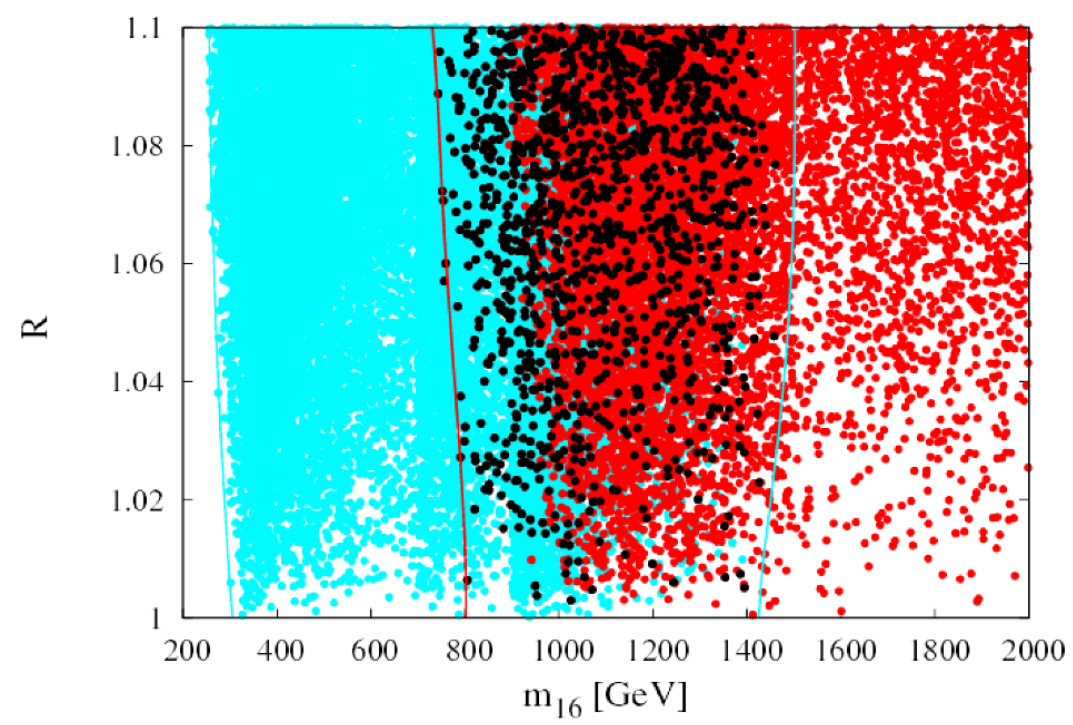

Figure 1. Plot of $R$ versus $m_{16}$. The regions inside the red (blue) contour are consistent with the constraint from $b \rightarrow s \gamma\left((g-2)_{\mu}\right)$. In the overlapping region of these contours $b \rightarrow s \gamma$ and $(g-2)_{\mu}$ can be satisfied simultaneously. The red (blue) points beside $b \rightarrow s \gamma\left((g-2)_{\mu}\right)$ satisfy the bound (6.6) for $\Omega_{D M} h^{2}$. The black points satisfy all the constraints including $b \rightarrow s \gamma,(g-2)_{\mu}$ and the upper WMAP bound.

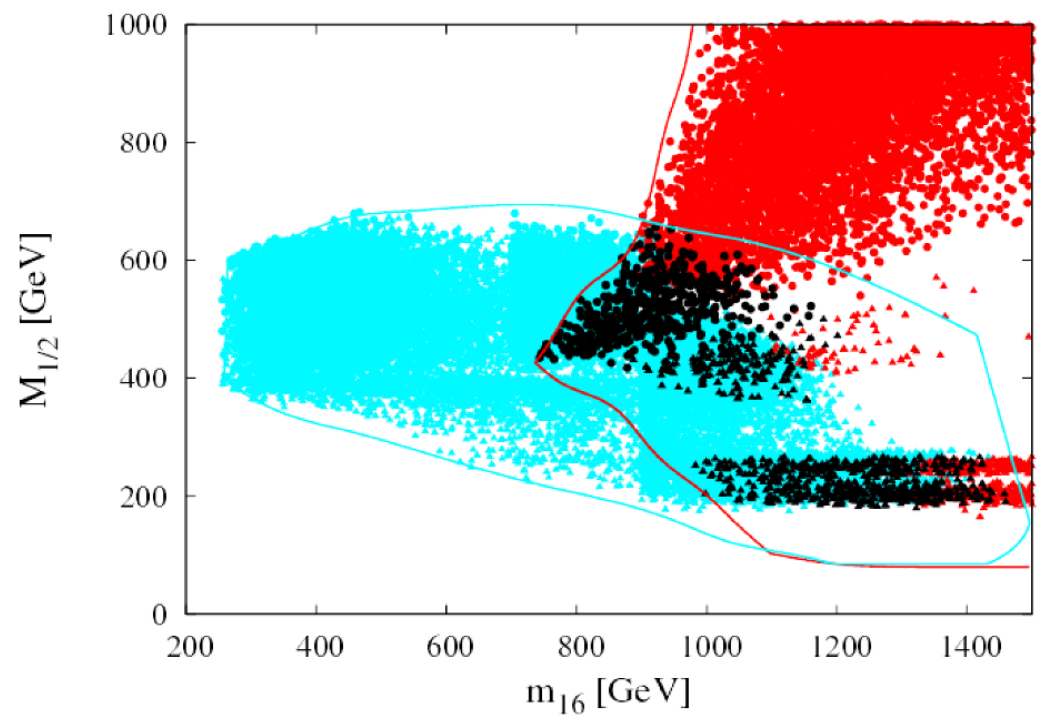

Figure 2. Plot of $M_{1 / 2}$ versus $m_{16}$ assuming $R \leqslant 1$.1. The key for the colors of the contours and the points is the same as in figure 1 . Triangles denote the points with $\left(m_{\tilde{\tau}}-m_{\mathrm{LSP}}\right) /\left(m_{\tilde{\tau}}+m_{\mathrm{LSP}}\right)>0.1$ i.e. solutions which satisfy WMAP bound without stau co-annihilations. 
This, however, implies also smaller values of $\left|\mu / M_{2}\right|$ which are disfavored by $b \rightarrow s \gamma$, as discussed in section 4.2. The tension between the constraints from $\Omega_{\mathrm{DM}} h^{2}$ and $b \rightarrow s \gamma,(6.6)$ and (6.4), results in a very small range of values of $M_{1 / 2}$ consistent with both observables.

For higher values of $M_{1 / 2}$ the Yukawa-unified solutions giving acceptable values of $(g-2)_{\mu}$ and $\mathrm{BR}(b \rightarrow s \gamma)$ satisfy the WMAP bound usually because of a rather high degree of degeneracy between the LSP and the stau which makes the co-annihilation with stau very efficient. This occurs for $M_{1 / 2}$ between about 400 and $650 \mathrm{GeV}$. In order to be compatible with the $\mathrm{BR}(b \rightarrow s \gamma)$ constraint one needs also $A_{0} / m_{16} \sim \mathcal{O}(2)$ to make the stop-mixing part of the chargino contribution to $b \rightarrow s \gamma$ negative, or at least strongly suppressed (see discussion in section 4.2). This is in contrast to the Yukawa-unified solutions which satisfy the WMAP bound due to the resonant annihilation through $Z$ or $h^{0}$ for which the stop-mixing part of the chargino contribution is suppressed because of a large hierarchy $M_{1 / 2} \ll m_{16}$ so the constraint on $\operatorname{BR}(b \rightarrow s \gamma)$ can be satisfied for any sign of $A_{0}$.

It can be also seen from figure 2 that there exist solutions consistent with the $(g-2)_{\mu}$ and $\mathrm{BR}(b \rightarrow s \gamma)$ bounds which satisfy the WMAP bound even without a quasi-degeneracy of the LSP and stau. They tend to have larger ratio $m_{16} / M_{1 / 2}$ and somewhat smaller values of $M_{1 / 2}$ than the solutions with the stau co-annihilation. These solutions can be divided into two subclasses. In the first one, the LSP annihilation through $A^{0}$ is efficient because $m_{A^{0}}$ is relatively light, with masses between about 300 and $400 \mathrm{GeV}$, while the LSP mass is about $100 \mathrm{GeV}$. It seems far away from the center of the $A^{0}$-resonance but this resonance is very broad because the coupling of $A^{0}$ to $b \bar{b}$ pairs is enhanced by large values of $\tan \beta \sim \mathcal{O}(50)$. Notice, however, that such a light $A^{0}$ implies that the contribution to $b \rightarrow s \gamma$ from the charged Higgs (which is almost degenerate with $A^{0}$ ) is very large and has to be (at least partially) canceled by a large negative chargino contribution. Such cancellation is possible because large positive values of $A_{0} / M_{1 / 2} \sim \mathcal{O}(5)$ lead to $A_{t}>0$ at the EW scale. Moreover, large values of $A_{0} / M_{1 / 2}$ drive stops masses to smaller values through RGEs. As a result, the chargino contribution to $b \rightarrow s \gamma$ is large and negative and may cancel the charged Higgs contribution. A large value of $A_{0} / M_{1 / 2} \sim \mathcal{O}(5)$ is also crucial for the second subclass of solutions consistent with all the constraints. Such large values of $A_{0} / M_{1 / 2}$ drive the mass of the right-handed sbottom (which is always the lightest squark due to the negative $D$-term contribution) to smaller values via the RG running of $m_{D}$. We found some Yukawa-unified solutions consistent with $(g-2)_{\mu}, \operatorname{BR}(b \rightarrow s \gamma)$ and DM relic abundance constraints with a very light sbottom, below about $200 \mathrm{GeV}$, for which the neutralinos annihilate very efficiently to $b \bar{b}$ pairs through the t-channel sbottom exchange. In such a case, there is no need for light $A^{0}$ nor for quasi-degeneracy between the stau and the LSP.

From the previous discussions it should be clear that large positive values of $A_{0}$ help to satisfy the experimental constraints. It is interesting to note, however, that there is an upper limit on $A_{0} / m_{16}$ even without taking the experimental constraints into account. The reason is as follows: Larger values of the $A$-terms increase the value of $\mu^{2}$ which, in turn, makes the sbottom-gluino contribution to the threshold correction to the bottom mass (see eq. (2.2)) more negative. Too large negative SUSY threshold correction to the bottom mass worsens bottom-tau Yukawa unification. We found numerically that Yukawa unification is impossible for $A_{0} \gtrsim 2.6 m_{16}$. 


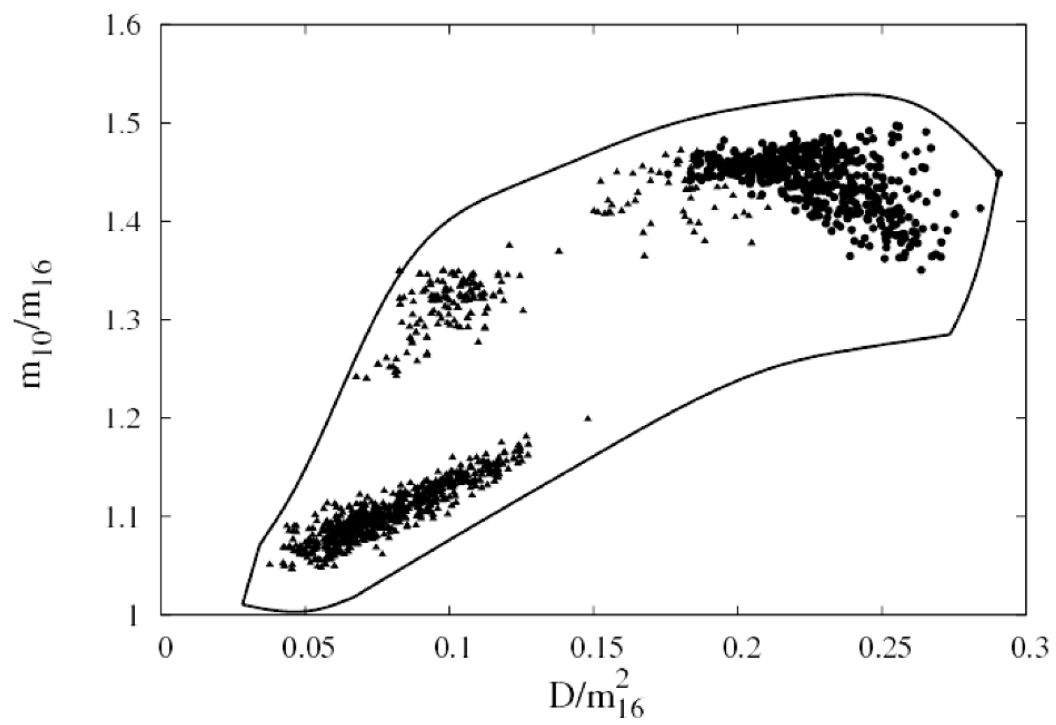

Figure 3. Plot of $m_{10} / m_{16}$ vs $D / m_{16}^{2}$ for solutions with $R \leqslant 1.1$ satisfying simultaneously $b \rightarrow s \gamma$ and $(g-2)_{\mu}$. The solutions satisfying WMAP bound are denoted by points while without imposing this bound they can be found anywhere inside the contour. Triangles denote the solutions with $\left(m_{\tilde{\tau}}-m_{\mathrm{LSP}}\right) /\left(m_{\tilde{\tau}}+m_{\mathrm{LSP}}\right)>0.1$.

We should also add that if one requires that only the $(g-2)_{\mu}$ bound (6.3) is satisfied but does not insist on fulfilling the bound (6.4) on $\mathrm{BR}(b \rightarrow s \gamma)$ the constraint on the $m_{16}-M_{1 / 2}$ plane from the relic abundance of neutralinos (6.6) is much weaker. Indeed, it can be seen from figure 2 that in such a case the values of $M_{1 / 2}$ from about $200 \mathrm{GeV}$ up to almost $700 \mathrm{GeV}$ may yield a correct relic abundance of neutralinos. The reason is that without imposing the $b \rightarrow s \gamma$ constraint the pseudoscalar Higgs $A^{0}$ can be very light (but not arbitrary light due to the constraint from $\operatorname{BR}\left(B_{s} \rightarrow \mu^{+} \mu^{-}\right)$) so the annihilation through $A^{0}$ exchange may be efficient enough for smaller masses of the LSP (corresponding to smaller values of $\left.M_{1 / 2}\right)$. However, there are no solutions satisfying the $(g-2)_{\mu}$ constraint with a correct relic abundance of the neutralinos for $M_{1 / 2} \gtrsim 300 \mathrm{GeV}$ and large values of $m_{16} \gtrsim 1300$ (the lower bound on $m_{16}$ decreases with increasing $M_{1 / 2}$ ).

One can infer from figure 3 that in order to have top-bottom-tau Yukawa unification the ratio $D / m_{16}^{2}$ has to be necessarily positive and larger than at least a few hundredths. It is also interesting to note that Yukawa unification seems to prefer $m_{10}>m_{16}$ because only $m_{10}>m_{16}$ may be consistent with $|\mu| \lesssim M_{1 / 2}$, as required for bottom-tau unification (unless $m_{\tilde{b}_{2}}>m_{\tilde{g}}$ ). Let us explain this point in more detail. At large $\tan \beta$, the condition of proper REWSB implies $\mu^{2} \approx-\left(m_{H_{u}}^{2}+M_{Z}^{2} / 2\right)$. Using this relation and the RGEs one can estimate electroweak scale value of $\mu^{2}$ in terms of the input parameters at the GUT scale:

$$
\mu^{2} \approx M_{1 / 2}^{2}\left[1.2+0.65 x^{2}\left(0.97-\frac{m_{10}^{2}}{m_{16}^{2}}+2.9 \frac{D}{m_{16}^{2}}+0.15 \frac{A_{0}^{2}}{m_{16}^{2}}-\frac{0.3}{x} \frac{A_{0}}{m_{16}}\right)\right]
$$


where $x \equiv m_{16} / M_{1 / 2}$. In order to satisfy the bound (2.4) the contribution from the gaugino masses to $\mu^{2}$ has to be (partially) canceled by other terms in (6.8). Yukawa unification requires positive $D$-terms so they give an additional positive contribution to $\mu^{2}$. Large values of $\left|A_{0}\right| / M_{1 / 2}$ also tend to make $\mu^{2} / M_{1 / 2}^{2}$ larger. So, partial cancellation in (6.8) may occur only for $m_{10}>m_{16}$. Since $\mu^{2}$ cannot be negative, Yukawa unification consistent with REWSB requires correlations among $m_{10}, D$ and $A_{0}$. These correlations are especially strong when $M_{1 / 2} \ll m_{16}$ because in such a case the value of $\mu^{2}$ is very sensitive to the value of the expression in the round bracket in eq. (6.8). The correlation between $m_{10} / m_{16}$ and $D / m_{16}^{2}$ is clearly visible in figure 3 .

\subsection{Predictions for the MSSM spectrum}

A great advantage of the assumption that top, bottom and tau Yukawa couplings unify at the GUT scale is its predictivity. When we supplement the condition of Yukawa unification by the requirement that $\mathrm{BR}(b \rightarrow s \gamma)$ and $(g-2)_{\mu}$ are consistent with the experimental data at the $2 \sigma$ level and the relic abundance of neutralinos agrees with observations, the predictions for the MSSM spectrum become even stronger. This follows mainly from the fact that in such a case the values of $M_{1 / 2}$ and $m_{16}$ are rather tightly constrained. The gluino mass is predicted to be between about 500 and $700 \mathrm{GeV}$ or $900 \mathrm{GeV}$ and $1.6 \mathrm{TeV}$. The gap is the consequence of the fact that there are no efficient LSP annihilation channels for values of $M_{1 / 2}$ corresponding to the gluino mass in range between about 700 and $900 \mathrm{GeV}$.

The predictions for other sparticles are also rather definite. The masses of the heavier stop and sbottom are predicted to be between about $800 \mathrm{GeV}$ and $1.4 \mathrm{TeV}$, while the lighter stop has mass typically below about $100-200 \mathrm{GeV}$. The right-handed sbottom is always lighter than other squarks because of the negative $D$-term contribution to its mass and typically has the mass between about 300 and $800 \mathrm{GeV}$. However, we have found solutions with the right-handed sbottom even as light as about $100 \mathrm{GeV}$. The squarks of the first two generations are typically $300-500 \mathrm{GeV}$ heavier than the corresponding squarks of the third generation because of negligible values of the corresponding Yukawa couplings.

Another prediction of our model is the mass of the lightest Higgs very close to the LEP2 bound. This is mainly a consequence of the fact that measured value of $(g-2)_{\mu}$ favors smaller values of $m_{16}$ which give lighter muon sneutrino but this results also in lighter stops making the radiative correction to the Higgs mass smaller. Moreover, suppressed stopmixing required by the $\mathrm{BR}(b \rightarrow s \gamma)$ results in an additional reduction of the Higgs mass. Nevertheless, as can be seen in figure 4 , the experimental bound on $(g-2)_{\mu}$ can be satisfied even at the $1 \sigma$ level without violating the constraint (6.4) on $b \rightarrow s \gamma$ if a conservative bound for the Higgs mass $m_{h}^{0}>111.4 \mathrm{GeV}$ is used (we recall that the theoretical uncertainty for the lightest CP-even Higgs mass is around $3 \mathrm{GeV})$. The maximal value of $(g-2)_{\mu}$ which may be obtained in our model drops down rather quickly with the Higgs boson mass but for $m_{h}^{0}>114 \mathrm{GeV}$ it can be still compatible with the experimental data at $2 \sigma$ level.

In figure 5 we present some characteristic spectra resulting from the assumption of top-bottom-tau Yukawa unification and consistency with all the experimental constraints including these on $\mathrm{BR}(b \rightarrow s \gamma)$ and $(g-2)_{\mu}$. Different spectra in figure 5 represent classes of solutions which satisfy the WMAP bound (6.6) due to different main annihilation channels 


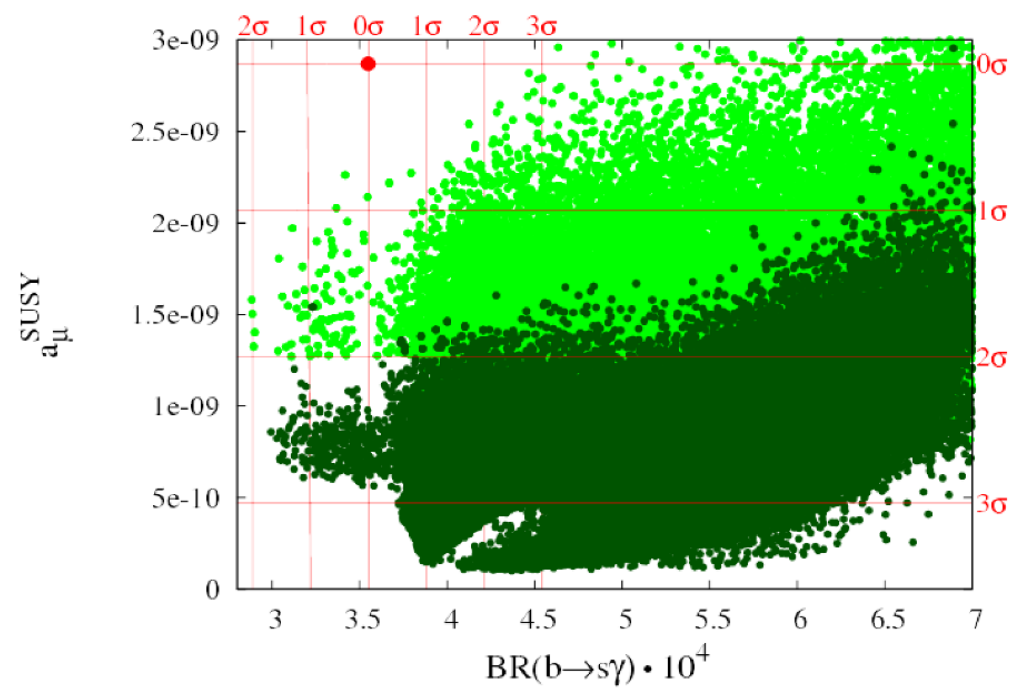

Figure 4. Plot of $a_{\mu}^{\mathrm{SUSY}}$ versus $\mathrm{BR}(b \rightarrow s \gamma)$ for the points with $R \leqslant 1.1$ and satisfying all the experimental constraints. For light (dark) green points $m_{h^{0}}>111.4\left(m_{h^{0}}>114\right) \mathrm{GeV}$.

of the LSP. Each spectrum has some characteristic features. In the case of the stau coannihilation there is a very large mass splitting within each generation of sfermions due to a large value of the $D$-term. In the case of the resonant annihilation through the $\mathrm{Z}$ boson, the gluino is very light. Moreover, since value of $\mu$ can be comparable with that of $M_{2}$ due to the hierarchy $M_{1 / 2} \ll m_{16}$ (see discussion in section 4.2) there is a smaller splitting in chargino sector and a larger mixing between wino and higgsino. This case differs from the others also because it has much smaller $\left|A_{0} / m_{16}\right|$ which results in a more compressed spectrum of the third generation. Moreover, the pattern of the slepton masses in this case reflects the $D$-term splitting at the GUT scale: the right-handed stau is heavier than the left-handed slepton doublet. On the other hand, in the case of the stau co-annihilation and light $A^{0}$, a large value of $A_{0} / m_{16}$ makes the left-handed doublet of the third generation sleptons heavier than the right-handed stau at the EW scale.

In table 1 the values of the relevant observables predicted by these benchmark points are presented. Notice that in each case the predicted relic density of neutralinos is within $3 \sigma$ from the WMAP central value.

We would like to emphasize that this is the first $\mathrm{SO}(10)$ model with top-bottom-tau Yukawa unification which predicts a light SUSY spectrum with all sparticle masses below $2 \mathrm{TeV}$ (in some cases even below $1.5 \mathrm{TeV}$ ) without violating any experimental constraints. This makes this model testable in the near future at the LHC. In fact, since the LHC is performing very well it may has already put some constraints on our model. The recent ATLAS analysis [37] set the lower bound on the gluino mass of about $750 \mathrm{GeV}$ in a simplified model consisting of gluino, degenerate squarks of the first two generations and the massless neutralino. However, this bound may be non-applicable to realistic models in which the squarks of the third generation must be present. It has been shown in [38] that the limits on 


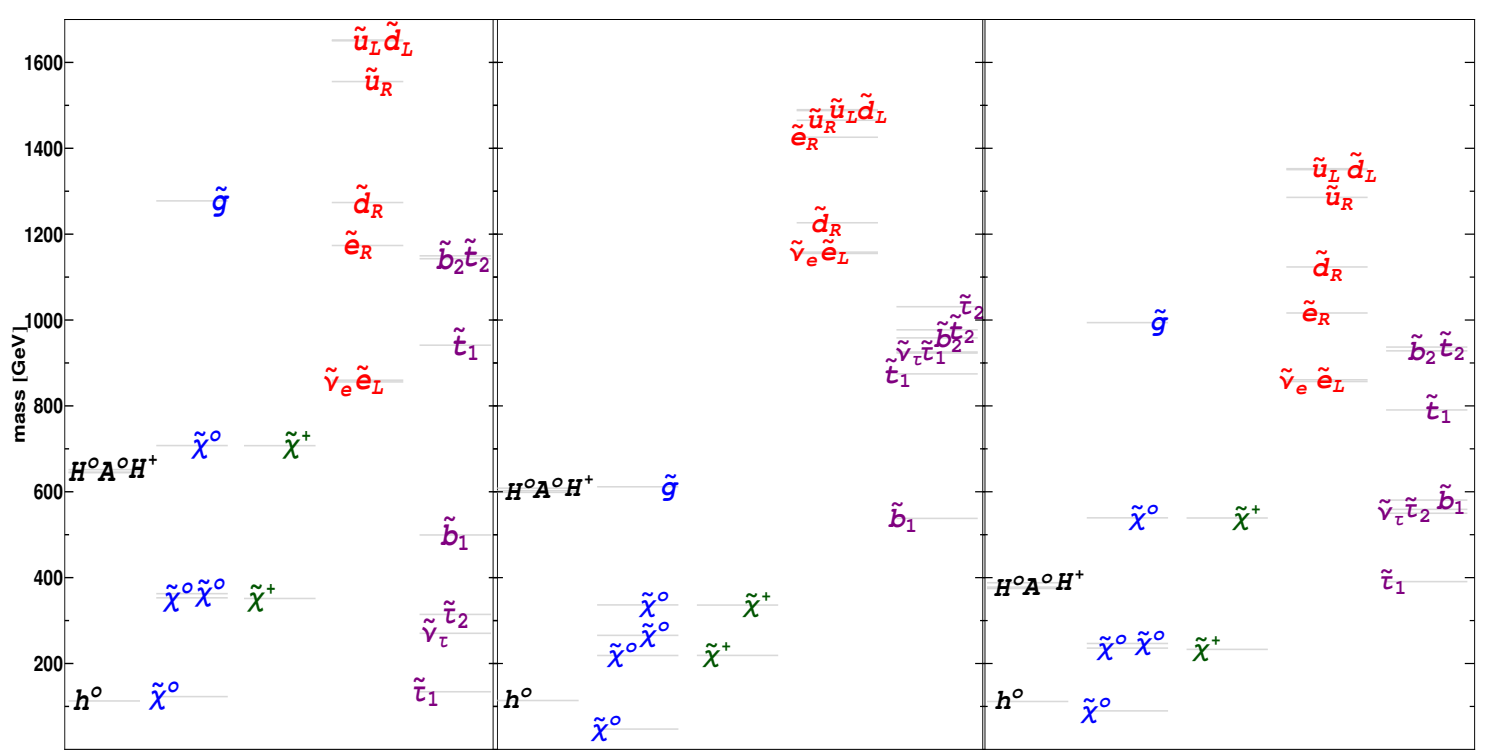

Figure 5. Typical spectra in the case of stau co-annihilation (left), resonant annihilation through the $\mathrm{Z}$ boson (centre) and the light $A^{0}$ (right). Sfermions of the second generation (not shown in the figure) are degenerate with the corresponding sfermions of the first generation. These spectra are obtained for the parameters presented in table 1 where also values of the relevant observables are given for each case.

\begin{tabular}{|l|c|c|c|}
\hline & left & centre & right \\
\hline$m_{16}$ & 1054 & 1352 & 949.5 \\
$M_{1 / 2}$ & 542.5 & 224.2 & 408.8 \\
$m_{10} / m_{16}$ & 1.454 & 1.125 & 1.324 \\
$D / m_{16}^{2}$ & 0.1907 & 0.0921 & 0.1154 \\
$A_{0} / m_{16}$ & 2.312 & -0.357 & 1.865 \\
$\tan \beta$ & 45.78 & 48.47 & 47.72 \\
\hline$a_{\mu}^{\mathrm{SUSY}}$ & $13.2 \times 10^{-10}$ & $14.2 \times 10^{-10}$ & $17.4 \times 10^{-10}$ \\
$\mathrm{BR}(b \rightarrow s \gamma)$ & $3.83 \times 10^{-4}$ & $4.11 \times 10^{-4}$ & $4.19 \times 10^{-4}$ \\
$\Omega_{\mathrm{DM}} h^{2}$ & 0.095 & 0.111 & 0.125 \\
\hline$R$ & 1.007 & 1.016 & 1.005 \\
\hline
\end{tabular}

Table 1. Input parameters corresponding to SUSY spectra presented in figure 5 and resulting values of the relevant observables.

the gluino mass from the ATLAS experiment are very weak in models with squarks of the third generation much lighter than these of the first two generations. In our model the EW scale intergenerational mass splitting of the squarks is rather significant, especially in the part of the parameter space predicting a light gluino between 500 and $700 \mathrm{GeV}$. Therefore, in order to set a firm exclusion limits on our model one needs a dedicated analysis which we leave for future work. 


\section{Conclusions}

We have investigated the SUSY SO(10) GUT model with the negative Higgs mixing parameter $\mu$. Non-universal soft SUSY breaking terms have been introduced but only those consistent with the $\mathrm{SO}(10)$ symmetry. Two sources of soft terms generating different masses within supermultiplets have been used: the D-terms associated with the breaking of $\mathrm{SO}(10)$ down to the SM gauge symmetry group in the scalar sector and $\mathrm{SO}(10)$ non-singlet $F$-terms transforming as the 24-dimensional representation of $\mathrm{SU}(5) \supset \mathrm{SO}(10)$ in the gaugino sector. No terms explicitly breaking $\mathrm{SO}(10)$, like e.g. an ad-hoc Higgs mass splitting, was allowed. We have shown that top-bottom-tau Yukawa unification in such a model is consistent with all experimental data. However, the present experimental bounds, especially those on $\operatorname{BR}(b \rightarrow s \gamma),(g-2)_{\mu}$ and the relic dark matter density, are strong and give an important constraints on the model parameter space. The main reason is some tension between the experimental results and the SM predictions for $\mathrm{BR}(b \rightarrow s \gamma)$ and $(g-2)_{\mu}$. The simplest way to fulfill the bound on $b \rightarrow s \gamma$ branching ratio is to have a heavy SUSY spectrum. On the other hand, the data on the muon anomalous magnetic moment prefer a light SUSY spectrum. The way to be consistent with the constraints on both quantities is to have relatively light sparticles but with some special features. This puts constraints on the parameter space of the model. The GUT scale value of the soft sfermion masses, $m_{16}$, must be in the $800-1400 \mathrm{GeV}$ range. The gluino mass parameter at the GUT scale, $M_{1 / 2}$, is between about 100 and $600 \mathrm{GeV}$ with precise bounds depending on $m_{16}$. The values of the remaining GUT scale soft parameters, $m_{10}, D$ and $A_{0}$, are correlated in order to obtain desirable values of the bottom mass threshold correction and of the chargino contribution to $b \rightarrow s \gamma$.

The LSP in our model is bino-like. There are several potentially important annihilation channels: the co-annihilation with stau, the resonance exchange of $Z$ boson or one of the Higgses ( $h^{0}$ or $A^{0}$ if it is light), the t-channel exchange of the lighter sbottom. The requirement that the LSP relic abundance is below the upper experimental limit restricts further the parameter space. There are two allowed ranges of $M_{1 / 2}$ consistent with all experimental constraints. They correspond to two ranges of the gluino mass: $500-700 \mathrm{GeV}$ and $900-1600 \mathrm{GeV}$. The lower range should be very soon tested in the LHC (the lower bound on the gluino mass of about $750 \mathrm{GeV}$, found by ATLAS collaboration in a simplified model, does not apply directly to our model). When the gluino mass is in the upper range, some other particles are relatively light with masses below $400 \mathrm{GeV}$. These may be the third generation sleptons (with the lighter stau nearly degenerated with the LSP neutralino), the lighter sbottom or the heavier Higgses. The LSP neutralino has a mass close to 45 or $55 \mathrm{GeV}$ (when it annihilates via the $Z$ or $h^{0}$ resonance) or between about 80 and $150 \mathrm{GeV}$ (when one of other annihilation channels is efficient). In addition, at least two other neutralinos and the lighter chargino have masses in the $200-400 \mathrm{GeV}$ range. Even the heaviest sparticles are lighter than $2 \mathrm{TeV}$ and quite often lighter than $1.5 \mathrm{TeV}$. Such SUSY spectrum is much lighter than in similar models with positive $\mu$. There are good chances that our model can be tested by the LHC experiments. 
Note added. During completion of this work ref. [39] appeared where top-bottom-tau Yukawa unification with $\mu<0$ and non-universal gaugino masses was investigated. Even though the same pattern of gaugino masses (3.4) was considered in [39] there is a major difference between our model and the one studied in [39]. Namely, in [39] an ad-hoc Higgs mass splitting is used which explicitly breaks $\mathrm{SO}(10)$ gauge symmetry. In the present paper we use the $D$-term splitting of the scalar masses which generically arises as a consequence of a spontaneous $\mathrm{SO}(10)$ symmetry breakdown.

\section{Acknowledgments}

This work has received funding from the European Community's Seventh Framework Programme under grant agreement PITN-GA-2009-237920 (2009-2013) and has been supported by MNiSzW scientific research grant N N202 103838 (2010-2012). MB would like to thank B. C. Allanach, G. Isidori, K. Sakurai and especially J. D. Wells for helpful and stimulating discussions.

Open Access. This article is distributed under the terms of the Creative Commons Attribution Noncommercial License which permits any noncommercial use, distribution, and reproduction in any medium, provided the original author(s) and source are credited.

\section{References}

[1] M.S. Carena, M. Olechowski, S. Pokorski and C.E.M. Wagner, Electroweak symmetry breaking and bottom - top Yukawa unification, Nucl. Phys. B 426 (1994) 269 [hep-ph/9402253] [SPIRES].

[2] M. Olechowski and S. Pokorski, Electroweak symmetry breaking with nonuniversal scalar soft terms and large tan beta solutions, Phys. Lett. B 344 (1995) 201 [hep-ph/9407404] [SPIRES].

[3] Y. Kawamura, H. Murayama and M. Yamaguchi, Low-energy effective Lagrangian in unified theories with nonuniversal supersymmetry breaking terms, Phys. Rev. D 51 (1995) 1337 [hep-ph/9406245] [SPIRES].

[4] H. Baer, M.A. Diaz, J. Ferrandis and X. Tata, Sparticle mass spectra from SO(10) grand unified models with Yukawa coupling unification, Phys. Rev. D 61 (2000) 111701 [hep-ph/9907211] [SPIRES].

[5] H. Baer et al., Yukawa unified supersymmetric SO(10) model: cosmology, rare decays and collider searches, Phys. Rev. D 63 (2000) 015007 [hep-ph/0005027] [SPIRES].

[6] S.P. Martin, Non-universal gaugino masses from non-singlet F-terms in non-minimal unified models, Phys. Rev. D 79 (2009) 095019 [arXiv:0903.3568] [SPIRES].

[7] T. Blazek, R. Dermisek and S. Raby, Yukawa unification in SO(10), Phys. Rev. D 65 (2002) 115004 [hep-ph/0201081] [SPIRES].

[8] D. Auto et al., Yukawa coupling unification in supersymmetric models, JHEP 06 (2003) 023 [hep-ph/0302155] [SPIRES].

[9] H. Baer, S. Kraml, S. Sekmen and H. Summy, Dark matter allowed scenarios for Yukawa-unified SO(10) SUSY GUTs, JHEP 03 (2008) 056 [arXiv:0801.1831] [SPIRES]. 
[10] H. Baer, S. Kraml and S. Sekmen, Is 'just-so' Higgs splitting needed for t-b-tau Yukawa unified SUSY GUTs?, JHEP 09 (2009) 005 [arXiv:0908.0134] [SPIRES].

[11] U. Chattopadhyay and P. Nath, $b$ - tau unification, $g(m u)$-2, the $b \rightarrow s+$ gamma constraint and nonuniversalities, Phys. Rev. D 65 (2002) 075009 [hep-ph/0110341] [SPIRES].

[12] U. Chattopadhyay, A. Corsetti and P. Nath, Supersymmetric dark matter and Yukawa unification, Phys. Rev. D 66 (2002) 035003 [hep-ph/0201001] [SPIRES].

[13] N. Chamoun, C.-S. Huang, C. Liu and X.-H. Wu, Non-universal gaugino masses in supersymmetric SO(10), Nucl. Phys. B 624 (2002) 81 [hep-ph/0110332] [SPIRES].

[14] I. Gogoladze, R. Khalid, S. Raza and Q. Shafi, t-b-tau Yukawa unification for $\mu<0$ with a sub-TeV sparticle spectrum, arXiv:1008.2765 [SPIRES].

[15] L.J. Hall, R. Rattazzi and U. Sarid, The top quark mass in supersymmetric SO(10) unification, Phys. Rev. D 50 (1994) 7048 [hep-ph/9306309] [SPIRES].

[16] D.M. Pierce, J.A. Bagger, K.T. Matchev and R.-j. Zhang, Precision corrections in the minimal supersymmetric standard model, Nucl. Phys. B 491 (1997) 3 [hep-ph/9606211] [SPIRES].

[17] K. Tobe and J.D. Wells, Revisiting top-bottom-tau Yukawa unification in supersymmetric grand unified theories, Nucl. Phys. B 663 (2003) 123 [hep-ph/0301015] [SPIRES].

[18] H. Murayama, M. Olechowski and S. Pokorski, Viable t-b- $\tau$ Yukawa unification in SUSY SO(10), Phys. Lett. B 371 (1996) 57 [hep-ph/9510327] [SPIRES].

[19] M. Davier, A. Hoecker, B. Malaescu and Z. Zhang, Reevaluation of the hadronic contributions to the muon g-2 and to alpha(MZ), Eur. Phys. J. C 71 (2011) 1515 [arXiv: 1010.4180] [SPIRES].

[20] Muon G-2 collaboration, G.W. Bennett et al., Final report of the muon E821 anomalous magnetic moment measurement at BNL, Phys. Rev. D 73 (2006) 072003 [hep-ex/0602035] [SPIRES].

[21] M. Misiak et al., The first estimate of $B\left(\right.$ anti- $B \rightarrow X / s$ gamma) at $O\left(\alpha_{s}^{2}\right)$, Phys. Rev. Lett. 98 (2007) 022002 [hep-ph/0609232] [SPIRES].

[22] Heavy Flavor Averaging Group collaboration, D. Asner et al., Averages of b-hadron, c-hadron and tau-lepton properties, arXiv:1010.1589 [SPIRES].

[23] P.H. Chankowski, J.R. Ellis, M. Olechowski and S. Pokorski, Haggling over the fine-tuning price of LEP, Nucl. Phys. B 544 (1999) 39 [hep-ph/9808275] [SPIRES].

[24] K.-i. Okumura and L. Roszkowski, Weakened constraints from $b \rightarrow s \gamma$ on supersymmetry flavor mixing due to next-to-leading-order corrections, Phys. Rev. Lett. 92 (2004) 161801 [hep-ph/0208101] [SPIRES].

[25] T. Moroi, The muon anomalous magnetic dipole moment in the minimal supersymmetric standard model, Phys. Rev. D 53 (1996) 6565 [hep-ph/9512396] [SPIRES].

[26] D. Stöckinger, The muon magnetic moment and supersymmetry, J. Phys. G 34 (2007) R45 [hep-ph/0609168] [SPIRES].

[27] G. Degrassi, P. Gambino and G.F. Giudice, $B \rightarrow X /$ s gamma in supersymmetry: large contributions beyond the leading order, JHEP 12 (2000) 009 [hep-ph/0009337] [SPIRES].

[28] H.E. Haber and G.L. Kane, The search for supersymmetry: probing physics beyond the standard model, Phys. Rept. 117 (1985) 75 [SPIRES]. 
[29] WMAP collaboration, E. Komatsu et al., Seven-year Wilkinson Microwave Anisotropy Probe (WMAP) observations: cosmological interpretation, Astrophys. J. Suppl. 192 (2011) 18 [arXiv: 1001.4538$]$ [SPIRES].

[30] N. Arkani-Hamed, A. Delgado and G.F. Giudice, The well-tempered neutralino, Nucl. Phys. B 741 (2006) 108 [hep-ph/0601041] [SPIRES].

[31] B.C. Allanach, SOFTSUSY: a program for calculating supersymmetric spectra, Comput. Phys. Commun. 143 (2002) 305 [hep-ph/0104145] [SPIRES].

[32] G. Bélanger, F. Boudjema, A. Pukhov and A. Semenov, MicrOMEGAs2.0: a program to calculate the relic density of dark matter in a generic model, Comput. Phys. Commun. 176 (2007) 367 [hep-ph/0607059] [SPIRES].

[33] Particle Data Group collaboration, K. Nakamura et al., Review of particle physics, J. Phys. G 37 (2010) 075021 [SPIRES].

[34] B.C. Allanach, A. Djouadi, J.L. Kneur, W. Porod and P. Slavich, Precise determination of the neutral Higgs boson masses in the MSSM, JHEP 09 (2004) 044 [hep-ph/0406166] [SPIRES].

[35] ALEPH collaboration, S. Schael et al., Search for neutral MSSM Higgs bosons at LEP, Eur. Phys. J. C 47 (2006) 547 [hep-ex/0602042] [SPIRES].

[36] CDF collaboration, T. Aaltonen et al., Search for $B_{s}^{0} \rightarrow \mu^{+} \mu^{-}$and $B_{d}^{0} \rightarrow \mu^{+} \mu^{-}$decays with 2ff ${ }^{-1}$ of p p collisions, Phys. Rev. Lett. 100 (2008) 101802 [arXiv:0712.1708] [SPIRES].

[37] ATLAS collaboration, Search for squarks and gluinos using final states with jets and missing transverse momentum with the ATLAS detector in $\sqrt{s}=7 \mathrm{TeV}$ proton-proton collisions, ATLAS-CONF-2011-086 (2011).

[38] K. Sakurai and K. Takayama, Constraint from recent ATLAS results to non-universal sfermion mass models and naturalness, arXiv:1106.3794 [SPIRES].

[39] I. Gogoladze, Q. Shafi and C.S. Un, SO(10) Yukawa Unification with $\mu<0$, arXiv:1107.1228 [SPIRES]. 